\title{
From Numerical to Symbolic Image Processing: Systems and Applications
}

\author{
Gianni Vernazza \\ Department of Biophysical and Electronic Engineering \\ Via Opera Pia 11 A \\ University of Genoa \\ I-16145 Genoa \\ Italy
}

\begin{abstract}
This June 1993 special section of Optical Engineering presents the innovative research and results on the integration between numerical and symbolic processing for image understanding. The integration of different techniques is regarded as the most advanced procedure for obtaining mutual advantages for overcoming individual limitations. From this standpoint, the symbolic approach (i.e., knowledge-based method) and numerical one (i.e., computational method) seem to be appropriate candidates for integration, because their functional advantages and structural limitations in the vision area are well known and have been widely experienced.
\end{abstract}

The computational approach is the most appropriate for performing lower level transformations; the knowledgebased approach is the most suitable for performing transformations and for representing data at higher levels (in particular, for contextual and semantic information).

An integrated knowledge-based computational system can also exploit the remarkable potential of artificial intelligence control techniques to manage processing tools and to apply backtracking mechanisms for error recovery.

Eight papers appear in this section covering various aspects of image understanding. Roli et al. discuss the control of both low-level and high-level phases of a multisensor image processing and recognition system based on a symbolic representation of control knowledge. Regazzoni, Foresti, and Murino propose a recognition system of planar surfaces by means of a hierarchical distributed vision system. A Bayesian belief network is used to propagate information between the different processing modules; local knowledge is used in addition to such constraints to regularize local problems. Binaghi and Rampini deal with the classification of multisource data, considered as a cognitive process, by using a knowledge-based approach. A fuzzy set representation framework is used to model this cognitive process. Bouthemy and Lalande describe an original framework to solve the problem of locating moving objects in a scene. It is based on a statistical regularization approach using spatiotemporal Markov fields, and it is independent of the size, intensity distribution, motion magnitude, and direction of the image of the moving objects. Li, Kittler, and Petrou develop a method of matching and recognizing aerial road network images based on road network models. Attribute relational graphs (ARGs) are used to describe images and models; the correspondences between image ARGs and model ARGs are found using a relaxation labeling algorithm. Del Bimbo, Landi, and Santini describe a neural system that performs learning and classification of 3-D planar-faced objects. The objects are described through a set of line descriptors; Kohonen networks with a nonmetric distance have been used to allow a humanlike classification of the objects views. Baujard and Garbay present a multiagent computer segmentation system (KISS). KISS demonstrates the applicability of a multiagent approach to computer vision. Segmentation is approached through a cooperative analysis involving both region and contour-based detection; interpretation of pattern is made using geometrical, relational, and semantic labeling. Bartolini et al. address the problem of evaluating a reliable optical flow field at motion boundaries. Velocity vectors are computed as solutions of a multiwindow least-squares problem. The field is then regularized by a vector median filter. The algorithm is noniterative and nonparametric.

I hope that these papers will contribute to a better picture of the different aspects related to the image understanding problem.

I would like to thank the authors who contributed the papers and the reviewers for their precious suggestions and time dedicated. Finally, I am grateful to Brian J. Thompson for giving me the opportunity of organizing this special section.

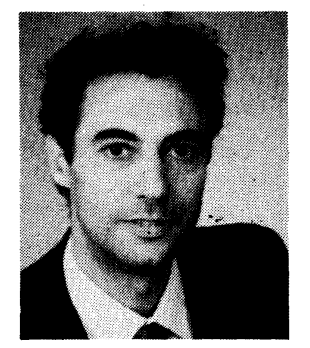

Gianni L. Vernazza is the manager of the Signal Processing and Understanding Group at the Department of Biophysical and Electronic Engineering (DIBE), University of Genoa, Italy. Dr. Vernazza received his laurea degree in electronic engineering from the University of Genoa in 1970. He then joined the Electrical Institute of the University of Genoa in 1976 as an assistant. Since 1982 he has been an associate professor of pattern recognition at DIBE. He has authored more than 150 technical publications in the area of signal processing. His current area of research is advanced signal processing, in particular, computer vision with recognition and understanding aspects by integrated methodologies (hybrid systems). 